\title{
Holomorphic Functions on Strict Inductive Limits of Banach Spaces
}

\author{
SEÁN DINEEN and LuIZA A. MORAES
}

\begin{abstract}
In this article we show that a number of apparently different properties coincide on the set of holomorphic functions on a strict inductive limit (all inductive limits are assumed to be countable and proper) of Banach spaces and that they are all satisfied only in the trivial case of a strict inductive limit of finite dimensional spaces. Thus the linear properties of a strict inductive limit of Banach spaces rarely translate themselves into holomorphic properties.
\end{abstract}

80

The main ingredients in our proof are the extension to strict inductive limits of a property of the $\tau_{\delta}$ semi-norms which was previously known for direct sums of Banach spaces [4], a characterization of Schwartz spaces using limited sets due to Lindström [8] and a close examination of a special hypoanalytic function on a strict inductive limit of Banach spaces constructed in [7].

We refer to [6] for details concerning holomorphic functions on locally convex spaces. Information regarding holomorphic functions on direct sums of Banach spaces, $\mathscr{D} \mathscr{F}$ spaces and on inductive limits of Fréchet spaces may be found in $[1,3,4,6,7,9]$. We thank J. M. Ansemil for some helpful comments.

\section{$\$ 1$}

In this section we recall some definitions and prove a number of results that we shall use later.

1991 Mathematics Subject Classification: $46 G 20$.

Editorial Complutense. Madrid, 1992. 
If $E$ is a locally convex space over $\mathbf{C}$ we let $\mathscr{Z}_{G}(E)$ denote the set of $\mathbf{C}$ valued functions on $E$ whose restriction to each finite dimensional subspace of $E$ is a holomorphic function (of several complex variables). If $f \in \mathbb{G}_{C}(E)$ is continuous on compact sets we say that $f$ is hypoanalytic and the space of all such functions is denoted by $\mathscr{Z}_{H Y}(E)$. If $f \in \mathscr{Z}_{G}(E)$ is continuous then $f$ is called holomorphic and we let $\mathscr{X}(E)$ denote the space of all holomorphic functions on $E$. We let $\mathscr{P}\left({ }^{n} E\right)$ denote the space of all continuous $n$ homogeneous polynomials on $E$.

The compact open topology on $\mathscr{E}(E)$ and $\mathbb{K}_{H Y}(E)$ is denoted by $\tau_{0 .}$. A semi-norm $p$ on $\mathscr{E}(E)$ is said to be $\tau_{\omega}$ continuous if there exists a compact subset $K$ of $E$ such that for each open subset $V$ of $E, K \subset V$, there exists $C(V)>0$ such that

$$
p(f) \leq C(V) \sup _{x \in V}|f(x)|
$$

for all $f \in \mathscr{Z}(E)$.

We, introduce the $\tau_{\delta}$ topology (see [6, proposition 3.27]) in a somewhat unusual fashion but one which is more suitable for the purposes of this article.

The $\tau_{\delta}$ topology on $\mathscr{H}(E)$ is the topology generated by all semi-norms which satisfy the following two conditions

$$
p(f)=\sum_{n=0}^{\infty} p\left(\frac{\hat{d}^{n} f(0)}{n !}\right)
$$

for every $f$ in $\$(E)$,

$$
p \mid \mathscr{p}\left({ }^{n} E\right)
$$

is $\tau_{\omega}$ continuous for each positive integer $n$.

If $\tau$ is a locally convex topology on $\mathscr{\mathscr { H }}(E)$, then $(\mathscr{W}(E), \tau)$ is T.S. $\tau$-complete if for any sequence $\left(P_{n}\right)_{n=0}^{\infty}, P_{n} \in\left({ }^{n} E\right)$ all $n$, such that $\sum_{n=0}^{\infty} p\left(P_{n}\right)<\infty$ for every $\tau$-continuous seminorm $p$ on $\mathscr{H}(E)$ we have $\sum_{n=0}^{\infty} P_{n} \in \mathscr{K}(E)([6$, definition
3.32]).

A subset $B$ of $E$ is said to be bounding if $\|f\|_{B}:=\sup _{\mathrm{r} \in B}|f(x)|<\infty$ for every $f \in \mathscr{H}(E)$ and $L \subset E$ is said to be limited if for every equicontinuous weak* null sequence in $E^{\prime},\left(\phi_{n}\right)_{n}$, we have $\left\|\phi_{n}\right\|_{L} \rightarrow 0$ as $n \rightarrow \infty$. 
Proposition 1. ([8]) A locally convex space E is a Schwartz space if and only if it is quasi-normable and the bounded subsets of $E$ are limited.

Proposition 2. If $E$ is a barrelled locally convex space then every weak* $n u l l$ sequence in $E^{\prime}$ converges uniformly to zero on the bounding subsets of $E$. In particular the bounding subsets of $E$ are limited.

Proof. Let $B$ denote a bounding subset of $E$ and let $\left(\phi_{n}\right)_{n=1}^{\infty}$ denote a weak* null sequence in $E^{\prime}$. Let $f=\sum_{n=1}^{\infty} \phi_{n^{n}}^{n}$ Since the sequence $\left(\phi_{n}\right)_{n}$ is weak* null the function $f$ is defined on all of $E$ and hence belongs to $\mathscr{H}_{G}(E)$. Since the sequence $\left(\phi_{n}\right)_{n}$ is pointwise bounded in the barrelled space $E$, it is equicontinuous. Thus $f$ belongs to $\mathscr{M}(E)$ by [2, proposition 1]. By corollary 4.19 (b) of [6] this implies

$$
\lim _{n \rightarrow \infty}\left(\left\|\phi_{n}^{n}\right\|_{B}\right)^{\frac{1}{n}}=\lim _{n \rightarrow \infty}\left\|\phi_{n}\right\|_{B}=0
$$

and hence $B$ is a limited subset of $E$. This completes the proof.

The following result is a reformulation of [7, proposition 3]. We sketch the proof for the readers' convenience

Proposition 3. Let $E=\lim _{\bar{n}} E_{n}$ denote a strict inductive limit of Banach spaces and suppose dimension $\left(E_{n}\right)=\infty$ for some integer $n$. Then there exists a $\mathrm{g}$ in $\mathrm{H}_{\mathrm{H}}(E)$ with the following properties;

$$
\begin{gathered}
g \notin \mathscr{L}(E) \\
\text { if } Z_{m}=\left\{n ;\left.\frac{\hat{d}^{n} g(0)}{n !}\right|_{E_{m}} \neq 0\right\} \\
\text { then } \sum_{n \in Z_{m}} \frac{\hat{d}^{n} g(0)}{n !} \in \mathscr{H}(E)
\end{gathered}
$$

for every positive integer $m$.

Proof. Since $\operatorname{dim}\left(E_{n}\right)=\infty$ for some $n, E$ is not a Schwartz space and hence proposition 1 implies that $E$ contains a bounded subset which is not limited. By proposition 2 the bounding subsets and the limited subsets of $E$ coincide and hence $E$ contains a bounded set, which we may suppose is the unit ball $B_{1}$ of $E_{1}$, which is not bounding. Hence there exists $f \in \mathscr{E}(E)$, 
$f=\sum_{n=1}^{\infty} P_{n}$, such that $\left\|P_{n_{j}}\right\|_{B_{1}} \geq 1$ for all $j$ where $\left(n_{j}\right)_{j}$ is a strictly increasing sequence of positive integers satisfying $n_{j+1}>n_{j}+j$ for all $j$.

Let $\phi_{n} \in E^{\prime}, n \geq 2$, satisfy $\phi_{n} \mid E_{n-1}=0$ and $\phi_{n}\left(x_{n}\right)=1$ for some $x_{n} \in E_{n}$ with $\left\|x_{n}\right\|=1$ and let $\theta_{k}(z)=k z$ for all $z \in E$.

We consider the function

$$
g=\sum_{k=2}^{\infty}\left\{\left(\sum_{j=k}^{\infty} P_{n_{j}}\right) \circ \theta_{k}\right\} \phi_{k}^{k}
$$

Since $j \geq k$ the condition $n_{j+1}>n_{j}+j$ implies that $\sum_{k=2, j \geq k}^{\infty}\left(P_{n j}{ }^{\circ} \theta_{k}\right) \phi_{k}^{k}$ is
Taylor series expansion of $g$.

Since

$$
g\left|E_{n}=\sum_{k=2}^{m+1}\left\{\sum_{j=k}^{\infty} P_{n j} \circ \theta_{k}\right\} \phi_{k}^{k}\right|_{E_{n}}
$$

it follows that $g \in \mathscr{H}_{H_{Y}}(E)$ and, moreover,

$$
\sum_{n \in Z_{m}} \frac{\hat{\hat{t}^{m}} g(\theta)}{n !} \in \mathscr{H}(E) \text { for all } m \text {. }
$$

We refer to [7] for the details which show that $g \notin \mathscr{H}(E)$.

Our next result was proved for countable direct sums of Banach spaces in [4] (see also [6, proposition 4.40]).

Proposition 4. If $E=\lim _{\vec{n}} E_{n}$ is a strict inductive limit of Banach spaces and $p$ is a $\tau_{\delta}$ continuous seminorm on $\mathscr{H}(E)$ then there exists a positive integer $m$ such that $f \in \mathscr{H}(E)$ and $f \mid E_{m}=0$ imply $p(f)=0$.

Proof. We may suppose without loss of generality that the semi-norm $p$ satisfies (1.1) and (1.2). If the result is not true then for every positive integer $n$ there exists a continuous homogeneous polynomial $P_{n}$ such that $P_{n} \mid E_{n}=0$ and $p\left(P_{n}\right) \neq 0$. We now show that the sequence $\left(P_{n}\right)_{n=1}^{\infty}$ is locally bounded.

Let $K$ denote a compact subset of $E$. Then $K$ is contained and compact in some $E_{k}$. For each positive integer $j$ let $B_{j}$ denote the unit ball in $E_{j}$.

Now choose $M>0$ and $\lambda_{1}, \ldots, \lambda_{k}$ positive numbers such that

$$
\left\|P_{j}\right\|_{K+\Sigma_{r=1}^{k} \lambda_{r} B_{r} \leq M} \text { for } j=1, \ldots, k
$$


Using a binomial expansion and the fact that $P_{k+1} \mid E_{k+1}=0$ we can find $\lambda_{k+1}>0$ such that

$$
\left\|P_{j}\right\|_{K+\sum_{r=1}^{k+1} \lambda_{r} B_{r}} \leq M+\frac{1}{2^{k+1}} \text { for } j=1 \ldots ., k+1 .
$$

By induction we can find a sequence of positive numbers $\left(\lambda_{r}\right)_{r=1}^{\infty}$ such that

$$
\left\|P_{j}\right\|_{K+\sum_{r=1}^{\infty} \lambda_{r} B_{r} \leq M+1 \text { for all } j .}
$$

Since $E$ is bornological it follows that $\sum_{r=1}^{\infty} \lambda_{r} B_{r}$ is a neighbourhood of zero in $E$. Hence $\left\{P_{j}\right\}_{j}$ is locally bounded.

If $Q_{j}=\frac{j P_{j}}{p\left(P_{j}\right)}$ then $Q_{j+\perp} \perp_{F_{j}}=0$ and the above argument shows that $\left\{Q_{j}\right\}_{j}$ is also a locally bounded and hence a $r_{\delta}$ bounded sequence in $\mathscr{H}(E)$ (see $[6$, lemma 2.43]).

Since $p\left(Q_{j}\right)=j$ this is impossible and proves our result.

Proposition 5. ([6, example 1.24]) If $E=\lim E_{n}$ is a inductive limit of Banach spaces then $\mathbf{C}$-valued homogeneous hypocontinuous polynomials on E are continuous.

We refer to [1], [3, proposition 4.1] and [6, example 1.38] for information regarding the next proposition.

Proposition 6. If $E=\lim _{\bar{n}} E_{n}$ is an inductive limit of Banach spaces then, for each positive integer $n, \bar{\tau}_{\omega}$ on $\mathscr{P}\left({ }^{n} E\right)$ is the topology of uniform convergence on the bounded subsets of $E$ and $\left(\mathscr{P}\left({ }^{n} E\right), \tau_{0}\right)$ and $\left(\mathscr{P}\left({ }^{n} E\right), \tau_{\omega \omega}\right)$ are complete locally convex spaces.

$\S 2$

The following theorem is our main result.

Theorem 7. If $E=\lim _{n} E_{n}$ is a strict inductive limit of Banach spaces then the following are equivalent:

(a) $\mathscr{Y}(E)=\mathscr{H}_{H Y}(E)$,

(b) the bounded subsets of $E$ are bounding, 
(c) the bounded subsets of $E$ are limited,

(d) $E \approx \mathrm{C}^{(M)}$,

(e) $\left(\mathscr{Z}(E), \tau_{0}\right)$ is a Fréchet space,

(f) $E=\lim _{\bar{n}} E_{n}$ in the category of locally convex spaces and holomorphic mappings,

(g) $\left(\mathscr{Y}(E), \tau_{0}\right)$ is complete (resp. quasicomplete, sequentially complete, T.S. $\tau_{0}$ complete),

(h) the $\tau_{0}$ bounded subsets of $\mathscr{H}(E)$ are locally bounded,

(i) $\left(\mathscr{H}(E), \tau_{\delta}\right)$ is complete (resp. quasicomplete, sequentially complete, T.S. $\tau_{\delta}$ complete),

(j) $\cdot \tau_{\delta}$ bounded subsets of $\mathscr{H}(E)$ are locally bounded.

Proof. The implication (a) $\Rightarrow$ (b) is proved in [7] (and also the implication (b) $\Rightarrow$ (a) if $E$ is separable). By proposition 2 we have (b) $\Rightarrow$ (c).

Since $E$ is a $\mathscr{Q} \mathscr{F}$ space it is quasinormable and hence if (c) is satisfied then proposition 1 implies that $E$ is a Schwartz space. Hence each $E_{n}$ is finite dimensional and (c) $\Rightarrow$ (d). By [5] condition (d) implies all the other conditions except (f). Since $C^{(M)}$ is a Schwartz space $(d) \Rightarrow(f)$. Hence conditions (a),..., (d) are all equivalent. By proposition 6 and $[6$, proposition 3.36] all the conditions in $(\mathrm{g})$ are equivalent, all the conditions in (i) are equivalent and $(\mathrm{e}) \Rightarrow(\mathrm{g}) \Rightarrow$ (i). If the $\tau$ bounded subsets of $\mathscr{K}(E)$ are locally bounded, $\tau=\tau_{0}$ or $\tau_{\delta}$, then we have $\mathscr{H}(E)$ T.S. $\tau$-complete, and hence $(\mathrm{h}) \Rightarrow(\mathrm{g})$ and $(\mathrm{j}) \Rightarrow$ (i). Hence, to complete the proof, it suffices to show $(\mathrm{f}) \Rightarrow(\mathrm{a})$ and $\sim(\mathrm{d}) \Rightarrow \sim$ (i). Suppose (f) is satisfied. Let $f \in \mathscr{X}_{H Y}(E)$. For each positive integer $n, f f_{E_{n}} \in \mathscr{I}_{H Y}\left(E_{n}\right)=\mathscr{H} /\left(E_{n}\right)$, since $E_{n}$ is a Banach space and the topology induced by $E$ on $E_{n}$ is its original Banach space topology. By (f) we have $f \in \mathscr{H}(E)$ and $(f) \Rightarrow($ a).

Now suppose that (d) is not satisfied. By proposition 3 there exists an $f=\sum_{n=0}^{\infty} \frac{\hat{d}^{n} f(0)}{n !}$ in $\mathscr{H}_{H Y}(E)$ satisfying (1.3) and (1.4). (1.2).

Let $p$ denote a $\tau_{\delta}$ continuous semi-norm on $\mathscr{H}(E)$ satisfying $(1.1)$ and

Let $m$ denote an integer associated with $p$ as in proposition 4 and let $Z_{m}$ have the meaning given to it in (1.4).

$$
\text { Since } \sum_{n \in Z_{m}} \frac{\hat{d}^{n} f(0)}{n !} \in \mathscr{H}(E) \text { we have } \sum_{n \in Z_{m}} p\left(\frac{\hat{d}^{n} f(0)}{n !}\right)<\infty .
$$


If $n \notin Z_{m}$ then $\left.\frac{\hat{d}^{n} f(0)}{n !}\right|_{t_{m}}=0$ and $p\left(\frac{\hat{d}^{n} f(0)}{n !}\right)=0$ by proposition 4 .

Hence $\sum_{n=0}^{\infty} p\left(\frac{\hat{d}^{n} f(0)}{n !}\right)<\infty$ and $\left\{\sum_{n=0}^{m} \frac{\hat{d}^{n} f(0)}{n !}\right\}_{m=1}^{\infty}$ is a Cauchy sequence in $\left(\mathscr{H}(E), \tau_{\delta}\right)$.

Since $\sum_{n=0}^{m} \frac{\hat{d}^{n} f(0)}{n^{n} !} \notin \mathscr{H}(E)$ this Cauchy sequence does not converge and (i) is not satisfied. This completes the proof.

Luiza A. Moraes would like to thank University College Dublin and CNPq (Brazil) for financial support to visit Dublin to undertake the research for this paper.

\section{References}

[1] J. M. ANSEMIL, S. PONTE: Topologies associated with the compact open topology on $\mathscr{H}(U)$, Proc. Royal Irish Acad., 82A, (1982), 121-128.

[2] M. BIANCHINI: $\mathscr{A}(E)$-bounded subsets of a locally convex space, Advances in Holomorphy, Ed. J. A. Barroso, North-Holland Math. Studies, 34, (1979), 103-110.

[3] J. Bonet, P. Galindo, D. Garcia, M. Maestre: Locally bounded sets of holomorphic mappings, T. A. M. S., 309, 2, (1988), 609-620,

[4] S. DINEEN: Holomorphic functions on a locally convex topological vector spaces, I: Locally convex topologies on $\$(U)$.. Ann. Inst. Fourier (Grenoble), 23, (1973), 19-54.

[5] S. DINEEN: Holomorphic functions on strong duals of Fréchet-Montel spaces, Infinite Dimensional Holomorphy and applications, Ed. M. C. Matos, NorthHolland Math. Studies, 12, (1977), 147-166.

[6] S. DINEEN: Complex Analysis in Locally Convex Spaces, North-Holland Math. Studies, 57, (1981).

[7] S. DineEN: Holomorphic functions on inductive limits of $\mathbf{C}^{N}$, Proc. Royal Irish Acad., A, 86, (1986), 143-146.

[8] M. Lindström: A characterisation of Schwartz spaces, Math. Z., 198, (1988), 423-430.

[9] L. A. MORAES: Holomorphic function on strict inductive limits, Res. Math. 4, (1981), 201-212.

Department of Mathematics,

University College Dublin,

Belfield, Dublin 4.

Ireland.
Instituto de Matemática Universidade Federal do Rio de Janeiro Caixa Postal 68530 21945 Rio de Janeiro

$$
\text { Brazil }
$$

Recibido: 2 de diciembre de 1991 Revisado: 22 de mayo de 1992 